\title{
A first assessment of the needs of young refugees arriving in Europe: what mental health professionals need to know
}

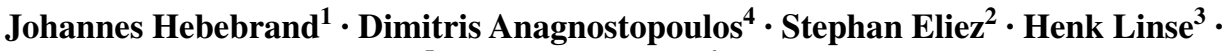 \\ Milica Pejovic-Milovancevic ${ }^{5} \cdot$ Henrikje Klasen $^{6}$
}

Published online: 22 December 2015

(C) Springer-Verlag Berlin Heidelberg 2015

Thousands of young refugees are currently entering Europe. They are exposed to many risks pre-flight, during their flight, and upon arrival, which make them vulnerable for the development of mental health problems. Our expertise as mental health professionals is crucial for the promotion of a healthy adaptation of these young people and their families and to lower their risks. In addition, it is important to identify young refugees with developing or preexisting serious mental disorders and to ensure access to evidence-based psychiatric treatment. The overarching aims of this ECAP editorial written by members of the ESCAP board representing different countries are to (1) exemplarily provide basic information on the dimensions of the crisis under special consideration of minors, illustrating that European countries are differentially affected, (2) provide an initial guide as to the needs of the young refugees during and after their flight,

(3) create a professional awareness of the implications of

Johannes Hebebrand

johannes.hebebrand@uni-due.de

1 Department of Child and Adolescent Psychiatry, Universitätsklinikum Essen, University of Duisburg-Essen, Wickenburgstr. 21, 45147 Essen, Germany

2 Département de l'instruction publique, de la culture et du sport (DIP), Office médico-pédagogique, Geneva, Switzerland

3 Anna Horstinkstraat, 36, 1382 MN, Weesp, Netherlands

4 Department of Child Psychiatry, General Pediatric Hospital of Athens "Aghia Sophia”, Medical School, National and Kapodistrian University of Athens, Athens, Greek

5 Department of Psychiatry, School of Medicine, University of Belgrade, Belgrade, Serbia

6 Associate Professor of Child Psychiatry, Leiden University Medical Centre, Leiden, Netherlands the current situation, (4) underscore the need for structured approaches toward acute and medium-term treatment, and (5) finally encourage us all to professionally deal with the crisis as cooperatively and creatively as we possibly can.

We are acutely aware of the fact that an assessment of the current situation is but a snapshot; undoubtedly, it will take time to enable child and adolescent psychiatrists and other mental health professionals throughout Europe to professionally achieve the aforementioned aims. We currently by no means have a sufficient insight into the different challenges that our colleagues face throughout Europe. The respective knowledge will grow regionally and nationally according to specific requirements; it is nevertheless important to bundle this growing information to provide overviews and to thus enable a more rapid, stringent, and targeted progress in the prevention and treatment of mental disorders. Currently, we are well aware of the fact that many questions cannot be answered yet in a satisfactory manner; as a first attempt to deal with such questions, we introduce an ESCAP online forum (http://www.escap.eu/care/organizing-the-knowledge-to-support-mental-health-care-for-refugee-children/) to openly discuss such issues and to allow expert articles to follow-up.

\section{The unfolding refugee crisis: every country has its own story to tell}

Flight and migration are not new phenomena, and many countries in Europe and the Middle East have been experiencing the recent refugee/migration wave (for the purpose of this editorial, we in the following refrained from distinguishing between immigrants and refugees) for several years. The number of refugees coming to Europe has currently reached staggering proportions in single countries; it is to some degree unpredictable to what extent and 
in what countries this influx will (intermittently?) increase or decrease. Only recently have some borders in Europe been opening up, thus relieving some of the pressure that has been building up in countries that have been exposed for a longer time period. In other countries, access of refugees has been curtailed substantially. In the second quarter of 2015, the five countries with the highest number of first-time asylum applications per 1,000,000 capita were Hungary ( $n=3,317)$, Austria (2026), Sweden (1467), Germany (997), and Malta (883). In all of Europe, first-time asylum applications increased by $15 \%$ between the first $(n=185,695)$ and second $(n=213,200)$ quarter of 2015 [8]. In EU-28, asylum applicants from Syria, Afghanistan, Eritrea, Kosovo, and Serbia predominated and accounted for $20,7,6,6$, and $5 \%$ of all applicants, respectively. The largest relative increase and decrease in 2014 as compared to 2013 were recorded for individuals from Ukraine and Russia, respectively.

Among the countries represented by ESCAP, the brunt of the refugee influx has been borne by those countries in southern, middle, and northern Europe that the refugees travel through and finally settle in. Turkey has given a provisional home to almost two million refugees, including 1.7 million Syrians (United Nations Health Commissioner for Refugees; UNHCR [29]). As conditions in the countries of origin and financial support of refugee camps deteriorated, more and more refugees have fled into different European countries following specific routes subject to change over time. Turkey, Greece, Malta, and Italy have been witnessing the influx or transit of refugees for the past few years. In Greece, for example, more than 710,000 refugees mainly stemming from Syria (69\%) and Afghanistan (21\%) have arrived by boat via this country's islands in 2015 . The associated risks are illustrated by the staggering number of 5000 shipwrecks in 2015; more than 89,000 immigrants and refugees were rescued [2]. Other countries such as Albania, Former Yugoslav Republic of Macedonia, Serbia [18], Hungary, Croatia, Austria, Germany, and Sweden have more recently begun to experience a major influx of refugees from Afghanistan, Iraq, Syria, and other Asian and African countries. Within EU-28, Germany has witnessed the largest increase in absolute numbers; the number of asylum applications increased steeply from 30,033 in 2009 to 362,153 in the first 10 months of 2015 [26]; the total number of refugees to arrive in this country in 2015 is estimated to be in the range of $1,000,000$.

\section{Minors represent one-fourth of all refugees}

In the EU-28, $26 \%$ of all asylum applicants in 2014 were minors $(19 \%<$ age 14; $7 \%$ between 14 and 17.9 years; [8]). Approximately 52 and $75 \%$ of the younger and older age groups were males. The vast majority ( $86 \%$ ) of migrant/refugee children travelled with their parents. The percentage of males was slightly over $50 \%$ for the accompanied versus over $85 \%$ for the unaccompanied. Despite (mandatory) medical examinations, verification of the age of a young refugee may prove impossible; depending on the perceived benefits in the respective country of arrival, age may either be falsely self-reported as younger or older. Age determinations via different methods are all imprecise and raise serious ethical questions for those involved [1, 11, 23].

The dramatic increase in the absolute number of unaccompanied minors merits consideration: For example, in Germany 6584 (5858 males) unaccompanied minors were taken into care by the youth welfare system in 2013; of these, 377 and 1647 were $<14$ years and between 14 and 16 years, respectively $[5,16]$. In 2015 , the total number of unaccompanied minors to have entered the country will definitely exceed 45,000 [6]; however, according to a reliable source (personal communication D. Göbel, LVR, Landesjugendamt) the number has currently (December $1,2015)$ exceeded 60,000 . It is obvious that this almost tenfold increase within just a short time period imposes a substantial burden on the German youth welfare system, entailing that considerable resources are being allocated to underage refugees. In the USA, information for child welfare personnel working with young refugees and their families has been compiled by Pine and Drachman [20].

\section{Synopsis of the situation of young refugees: what to consider}

Obviously, the pre-flight or pre-migration experiences of young refugees depend on their country of origin; exposures to poverty, war, or war-like conditions are common. In addition, the acquired education, social status, familial, religious, and sociocultural values also shape coping and help-seeking behavior. Healthcare professionals need to take into account that the citizenships of refugees differ substantially according to the respective European country, in which they apply for asylum (for details, see Eurostat [8]).

In addition to the potentially traumatic experiences in the countries of origin, an understanding of the flight experience of the individual refugee is also critically important for planning mental health services in the post-flight context, as the flight in itself can be traumatic or compound trauma via, for instance, separation experiences, sexual abuse (see Office of Refugee Resettlement et al. [17]), and trafficking including forced labor and sexual exploitation. In this context, it should be pointed out that many unaccompanied underage refugees attempt to travel in groups, 
which include adults known to them; in many cases, the parents of unaccompanied children and adolescents are aware of their travels. To allow for better protection of such young refugees, it would seem helpful to advise authorities not to totally separate underage refugees from such groups.

Finally, the arrival in the hosting country entails risks due to unsafe or otherwise problematic living conditions, non-access to schooling, years of insecurity with uncertain status, multiple moves, parental illness and unemployment, social exclusion, and in the medium- and long-term maladaptation with respect to the cultural norms of the hosting country. Hostility to foreigners and refugees in particular represents a threat that requires both surveillance of refugee camps and political education within the hosting country. Within this context, the initial provision of a safe environment to traumatized young refugees should not be taken for granted.

\section{Physical health issues predominate upon arrival}

After reaching the hosting country, the refugees are physically exhausted; at this stage, provision of somatic health care represents a major concern. For instance, in Serbia common acute health problems have included middle ear infections, angina, bronchitis, sinusitis, varicella, pneumonia, laryngitis, dermatological conditions, dehydration, blisters and diarrhea; infrequently, previously poorly treated children with congenital malformations and other serious somatic/neurological disorders pose significant challenges to the national healthcare system [18]. Similarly, a German cross-sectional survey based on 102 unaccompanied asylum-seeking adolescents aged 12-18 years revealed a complex disease burden of physical disorders with a high prevalence of infections $(58.8 \% ; 20 \%$ with parasites), iron deficiency anemia (17.6\%), and a very low prevalence of non-communicable diseases $(<2.0 \%$; [14]). The high prevalence of physical and in particular communicable disorders is echoed in a review of the health status of adolescent refugees who are resettling in high-income countries [10]. In addition, nutritional deficiencies warrant attention.

\section{Mental health problems as reported in studies published in 2015}

A cursorily performed PubMed search on articles published in 2015 on child and adolescent refugees provides some information relevant for the current situation in Europe. However, knowledge is limited and fragmented, because populations of different backgrounds have been assessed; the total number of publications is quite limited.
Only single studies of higher quality were detected; case numbers in original reports were frequently low; the methodology employed was frequently rather crude. Accordingly, the generalizability of such results must be viewed with considerable caution.

In the aforementioned German survey [14], mental illness was observed in $13.7 \%$. Overall, females were more frequently affected. The elevated risk of females was also apparent in a review of 17 Canadian studies based on young Canadian refugees [9], which assessed determinants of mental illness such as pre-migration experiences, number of years since immigration to Canada, post-migration family and school environment, in- and out-group problems, discrimination, and lack of equitable access to health care. Some of the included studies identified fewer emotional and behavioral problems among refugee youth; others reported higher rates of psychopathology as compared with Canadian-born youths. Pre-migration experiences and the kinds of trauma experienced proved to be relevant for the mental health outcomes. The risk to develop mental health problems proved to be elevated, particularly among unaccompanied refugees and in those with experiences in detention. Educational, acculturational, legal, and ethical issues in addition to socioeconomic factors also influenced the risk. Evidence for the importance of family involvement, school settings as points of care and services, and a focus on the first year of arrival was also detected. Themes of cultural belongingness and identification, psychological functioning, family unit functioning and relationships, and friendships and interpersonal processes are important for young refugees [15].

In Malmö, Sweden, unaccompanied minors, most of them males from Afghanistan, were shown to be overrepresented in psychiatric inpatient care [22]: $3.4 \%$ of unaccompanied minors in contrast to $0.26 \%$ of other minors of the catchment area received inpatient treatment. A large percentage ( $86 \%$ out of $n=52$ ) was admitted with symptoms related to stress in the asylum process. Neurotic disorders (ICD-10 F40-48) were more common in the unaccompanied refugees. The long-lasting and dire implications of displacement of children, who unlike adults are unable to communicate their needs and traumatic experiences, were reviewed by Joshi and Fayyad [12].

Sleijpen et al. [25] in a review of 26 studies identified the following six sources of resilience, most of which also had counterproductive aspects, in young refugees, who had resettled in Western countries: social support, acculturation strategies, education, religion, avoidance, and hope. Resilience assessed 20 years after displacement of Sri Lankans proved to be more strongly and robustly associated with economic and social factors than with the presence of mental disorders. 
Refugees may hold negative attitudes toward mental health and distrust services as shown in a study encompassing 15 young refugees, potentially reflecting their experiences within their home country of psychiatric care, their experiences of being a refugee/asylum-seeker and/or cultural differences [13].

\section{Public relations activities}

Undoubtedly, the term refugee crisis is warranted to describe the flight of so many people from their native countries and the associated personal and societal turmoil. At the European level, we are also witnessing an ethical crisis, in that several countries for whatever reasons do not want refugees to enter their countries; we should avoid prejudice based on our own personal view; it is important to constructively interact with colleagues from other countries, whose policies may differ substantially from those of our own country. Overall, as child and adolescent psychiatrists we should stress the fact that approximately $25 \%$ of refugees are children and adolescents. It is quite obvious that in most cases the respective parents or other caregivers choose to send these children on their way despite the well-known substantial risks associated with the flight including death and the uncertainties pertaining to the future of these young individuals in the country in which they finally arrive including the potential possibility that they will be deported. At the national level, the specific challenges and opportunities for child mental health differ across Europe depending on the number of refugees, their countries of origin and their age. Using our expertise and showing leadership in this situation are important and can prevent suffering now and in the future. In some countries (see Anagnostopoulos et al. [2]), the term refugee crisis represents an understatement and adds to already existent severe economic hardships. In comparison, the use of the same term appears to be an overstatement in other countries, which have either not been substantially exposed to a refugee influx or who in comparison with countries such as Greece have much better financial resources to cope with the influx. Depending on country of origin, we thus deem it important to critically reflect on the term crisis or other alarmistic wording to describe the current situation; the way we speak of the refugees and the migration wave has important connotations for the general public and particularly for the professionals with whom we work together to alleviate the difficult situation of the young refugees. We need to be aware of our responsibility when we are involved with public media; our wording can be instrumentalized. A look across borders into other European countries is helpful for a critical self-evaluation of the problems related to the refugee influx within one's own country. On a worldwide basis, Europe only hosts a rather small minority of all refugees, whose count was 51.2 million in 2014 (50\% aged $<18$ years; UNHCR [30]).
We should realize that financial resources of every country hosting a larger number of refugees are strained. If questioned, we should advice politicians as to how to make optimal use of funding. Investments are initially primarily required for provision of a safe environment, appropriate schooling, and the youth welfare system. Because many refugees do not speak our language and need to acculturate, it will frequently take 1-2 years until they establish solid contacts with mental health services. We should use this time span to structure our help as best as possible.

\section{Outline of the work ahead of us}

We need to make ourselves familiar with the mental health issues of refugees. The WHO published information on the rapid assessment of mental health needs of refugees, displaced and other populations affected by conflict and post-conflict situations [19]. An excellent informative Web site available in both English and French is the Refugee Mental Health Project of Canada's Mental Health and Addiction Network [7]. The up-to-date Web site provides access to: two self-directed online courses, community of practice, toolkit of resources, webinar series, and networking events. The toolkit includes a link to "Evidence-based clinical guidelines for immigrants and refugees" published in the Canadian Medical Association Journal in 2011 ([21]; see also [28]). Witt et al. [31] provided an overview of the mental disorders of unaccompanied young refugees. For this purpose, 43 articles were systematically identified and reviewed. The acute psychosocial interventions for traumatized refugee children and families have been delineated by Brymer et al. [4].

Our current structures, healthcare financing, and ways of working are often focussed on the individual or families. However, to meet the challenge we might need to adopt a more public health approach making more use of screening, stepped care, task sharing, and task shifting-methods that have been developed in global mental health and might be useful in Europe, too. Importantly, we need to teach mental healthcare professionals including teachers and social workers, who can incorporate our approach in their daily work.

The acute needs of child and adolescent psychiatry should follow the different needs and peculiarities that every country is faced with concerning the refugee crisis under consideration of the different stages of the refugee flight and resettlement process. Communication is facilitated by ESCAP through the use of the Web site and ESCAP communication pages or the journal European Child + Adolescent Psychiatry (ECAP). This communication will prove vital to speed up the efforts to competently deal with mental health problems of young refugees. As 
such, we require international meetings to exchange our knowledge.

In daily interaction with refugees as patients and their caregivers, language and cultural problems arise frequently and access to regular, expert interpreting services is limited. Further employment and training of interpreters and bicultural workers and collaboration with the local cultural groups are necessitated. Also, practitioners may enhance their cultural competence through relevant knowledge accessible through refugee-specific websites. For instance, the Cultural Orientation Resource Center (http://www.cal. $\mathrm{org} / \mathrm{co} /$ ) has compiled culture profiles, which provide a basic introduction to the social structure, language, geography, and history of various cultural groups. Also, we should reconsider our familiar models of psychotherapy in order to accommodate the possibly different meanings of mental health, trauma, and support in the refugees' cultural context. Internet Web sites can also be a very useful tool for enhancing cultural competence of professionals and for monitoring refugees' access and utilization of services. Moreover, refugees themselves may use such applications in order to get information, access facilitation, and be aware of new actions tailored to their needs (e.g., http:// www.healthgate4all.gr/).

Variability concerning observed mental disorders may be attributable to limitations of cultural applicability of the "trauma model" and other Western methods of mental health assessment in non-Western populations (e.g., [3, 27]. Maybe new diverse conceptual frameworks for understanding the refugee reactions and adjustment are needed. Except for mental disorders, clinicians should give a special emphasis toward understanding refugees' experiences and challenges within the new environment and toward fostering resilience among individuals and communities.

\section{The role of ESCAP}

We want to support professionals and are therefore inviting everyone to contribute to an online forum (http://www. escap.eu/care/organizing-the-knowledge-to-support-mental-health-care-for-refugee-children/) to share their experience and questions. We will gather issues from caregivers who have worked with refugee children, making use of our Europe-wide network of ESCAP members, and from the refugee communities themselves which we will address through our clinical network and partner organizations. Urgent questions will be addressed by matching experts from our scientific network of ESCAP Field Advisors, and the issues raised in the Forum will be processed by the Guidance Group in the ESCAP Clinical Division with consultation of all relevant experts from the ESCAP network to produce an evidence-based guidance for child and adolescent mental health in refugees and migrants. Several instruments for different audiences will be distributed within the next 6 months, making the guidance available for: (a) a broad audience (all mental health workers and non-professional volunteers), (b) psychologists, social workers, and (c) child and adolescent psychiatric services.

ESCAP should take an initiative to call for a joint research project, which mainly focusses on the acute needs of refugees, the risk and protective factors for their mental health, and the specific interventions that are needed, taking into consideration the different needs and available resources of the various EU countries. Such an initiative should be started with an open invitation to all our members to participate and should be the basis for advocating to health policy makers.

European attitude toward young refugees and their families will greatly determine the burden of trauma and attachment disorders on their adult future but also on our community. Indeed, the abundant literature of clinical and neuroscience research from the last 15 years proves undoubtedly that the impact of chronic stress and attachment disorder expands over at least two generations. Studies demonstrated the increased rate of personality disorders, chronic depression, conduct disorders, substance use disorders, lower education, higher unemployment, and social marginalization among individuals that have experienced early trauma and subsequent attachment disorders. Conversely, we know that an empathic and mentalizing attitude, secure sheltering, addressing health and educational needs will create a sense of stability and confidence. This is the very first step to favor, for these future adults and their family, either a productive integration in the European heritage of strength and diversity, or the potential to rebuild and stabilize their native countries for those who will return.

\section{References}

1. Abbing HD (2011) Age determination of unaccompanied asylum seeking minors in the European Union: a health law perspective. Eur J Health Law 18(1):11-25

2. Anagnostopoulos DC, Triantafyllou K, Xylouris G, Bakatsellos J, Giannakopoulos G (2016) Communications of the European Society for Child and Adolescent Psychiatry. Eur Child Adolesc Psychiatry. doi:10.1007/s00787-015-0806-1

3. Bracken PJ (2002) Trauma: culture, meaning and philosophy. Whurr Publishers, London

4. Brymer MJ, Steinberg AM, Sornborger J, Layne CM, Pynoos RS (2008) Acute interventions for refugee children and families. Child Adolesc Psychiatr Clin N Am 17(3):625-640, ix. doi:10.1016/j.chc.2008.02.007

5. Bundestag (2015) https://www.bundestag.de/presse/hib/2015_08/ $-/ 384870$

6. Bundesverband unbegleitete minderjährige Flüchtlinge. http:// www.b-umf.de/ 
7. Canada's Mental Health \& Addiction Network. Refugee Mental Health Project. https://www.porticonetwork.ca/web/rmhp/home

8. Eurostat. http://ec.europa.eu/eurostat/statistics-explained/index. php/Asylum_statistics

9. Guruge S, Butt H (2015) A scoping review of mental health issues and concerns among immigrant and refugee youth in Canada: looking back, moving forward. Can J Public Health 106(2):e72-e78. doi:10.17269/cjph.106.4588

10. Hirani K, Payne D, Mutch R, Cherian S (2015) Health of adolescent refugees resettling in high-income countries. Arch Dis Child. doi:10.1136/archdischild-2014-307221 (Epub ahead of print)

11. Hjern A, Brendler-Lindqvist M, Norredam M (2012) Age assessment of young asylum seekers. Acta Paediatr 101(1):4-7. doi:10.1111/j.1651-2227.2011.02476.x (Epub 2011 Oct 20)

12. Joshi PT, Fayyad JA (2015) Displaced children: the psychological implications. Child Adolesc Psychiatr Clin N Am 24(4):715730. doi:10.1016/j.chc.2015.06.003 (Epub 2015 Jul 3)

13. Majumder P, O'Reilly M, Karim K, Vostanis P (2015) This doctor, I not trust him, I'm not safe': the perceptions of mental health and services by unaccompanied refugee adolescents. Int J Soc Psychiatry 61(2):129-136. doi:10.1177/0020764014537236 (Epub 2014 Jun 3)

14. Marquardt L, Krämer A, Fischer F, Prüfer-Krämer L (2015) Health status and disease burden of unaccompanied asylumseeking adolescents in Bielefeld, Germany: cross-sectional pilot study. Trop Med Int Health. doi:10.1111/tmi.12649 (Epub ahead of print)

15. McGregor LS, Melvin GA, Newman LK (2015) Differential accounts of refugee and resettlement experiences in youth with high and low levels of posttraumatic stress disorder (PTSD) symptomatology: a mixed-methods investigation. Am J Orthopsychiatry 85(4):371-381. doi:10.1037/ort0000076

16. Müller A (2014) Unbegleitete Minderjährige in Deutschland, working paper 60 des Forschungszentrums des Bundesamtes. Nürnberg: Bundesamt für Migration und Flüchtlinge. https:// www.bamf.de/SharedDocs/Anlagen/DE/Publikationen/EMN/ Nationale-Studien-WorkingPaper/emn-wp60-minderjaehrige-indeutschland.pdf?_blob=publicationFile

17. Office of Refugee Resettlement (ORR), Administration for Children and Families (ACF), Department of Health and Human Services (HHS) (2014) Standards to prevent, detect, and respond to sexual abuse and sexual harassment involving unaccompanied children. Interim final rule (IFR). Fed Regist 79(247):77767-77800

18. Pejovic-Milovancevic M (2015) http://www.escap.eu/bestanden/ Care\%20(38)/Refugees/serbia_perspective_25112015.pdf

19. Petevi M, Pierre Revel J, Jacobs GA (2001) Rapid assessment of mental health needs of refugees, displaced and other populations affected by conflict and post-conflict situations and available resources. http://www.who.int/hac/techguidance/pht/7405.pdf

20. Pine BA, Drachman D (2005) Effective child welfare practice with immigrant and refugee children and their families. Child Welfare 84(5):537-562

21. Pottie K, Greenaway C, Feightner J, Welch V, Swinkels H, Rashid M, Narasiah L, Kirmayer LJ, Ueffing E, MacDonald NE, Hassan G, McNally M, Khan K, Buhrmann R, Dunn S, Dominic A, McCarthy AE, Gagnon AJ, Rousseau C, Tugwell P, Coauthors of the Canadian Collaboration for Immigrant and Refugee Health (2011) Evidence-based clinical guidelines for immigrants and refugees. CMAJ 183(12):E824-E925. doi:10.1503/ cmaj.090313 (Epub 2010 Jun 7)

22. Ramel B, Täljemark J, Lindgren A, Johansson BA (2015) Overrepresentation of unaccompanied refugee minors in inpatient psychiatric care, vol 4. Springerplus, Berlin, p 131. doi:10.1186/ s40064-015-0902-1 (eCollection 2015)

23. Sauer PJ, Nicholson A, Neubauer D, Ethics Group of the European Academy of Paediatrics (2015) Advocacy and age determination in asylum seekers: physicians should not be implicated. Eur J Pediatr (Epub ahead of print)

24. Siriwardhana C, Abas M, Siribaddana S, Sumathipala A, Stewart $\mathrm{R}$ (2015) Dynamics of resilience in forced migration: a 1-year follow-up study of longitudinal associations with mental health in a conflict-affected, ethnic Muslim population. BMJ Open 5(2):e006000. doi:10.1136/bmjopen-2014-006000

25. Sleijpen M, Boeije HR, Kleber RJ, Mooren T (2015) Between power and powerlessness: a meta-ethnography of sources of resilience in young refugees. Ethn Health. 2015:1-23. doi:10. 1080/13557858.2015.1080484

26. Statista. http://de.statista.com/statistik/daten/studie/76095/umfrage/ asylantraege-insgesamt-in-deutschland-seit-1995/

27. Summerfield D (1999) A critique of seven assumptions behind psychological trauma programmes in war-affected areas. Soc Sci Med 48:1449-1462

28. Swinkels H, Pottie K, Tugwell P, Rashid M, Narasiah L, Canadian Collaboration for Immigrant and Refugee Health (CCIRH) (2011) Development of guidelines for recently arrived immigrants and refugees to Canada: Delphi consensus on selecting preventable and treatable conditions. CMAJ 183(12): E928E932 http://www.ncbi.nlm.nih.gov/pmc/articles/PMC3168668/

29. United Nations Health Commissioner for Refugees. http://www. unhcr.org/pages/49e48e0fa7f.html

30. United Nations High Commissioner for Refugees (UNHCR) (2014) Global Trends 2013. UNHCR, Genf

31. Witt A, Rassenhofer M, Fegert JM, Plener PL (2015) Hilfebedarf und Hilfsangebote in der Versorgung von unbegleiteten minderjährigen Flüchtlingen. Eine systematische Übersicht. Kindheit und Entwicklung 24(4):209-224 